\title{
Deep Drilling and Sampling via the Wireline Auto-Gopher Driven by Piezoelectric Percussive Actuator and EM Rotary Motor
}

\author{
Yoseph Bar-Cohen ${ }^{1}$, Mircea Badescu ${ }^{1}$, Stewart Sherrit ${ }^{1}$, Kris Zacny ${ }^{2}$, Gale L Paulsen ${ }^{2}$, Luther \\ Beegle $^{1}$, Xiaoqi Bao ${ }^{1}$ \\ ${ }^{1}$ Jet Propulsion Laboratory, California Institute of Technology, Pasadena, CA \\ ${ }^{2}$ Honeybee Robotics Spacecraft Mechanisms Corporation, Pasadena, CA
}

\begin{abstract}
The ability to penetrate subsurfaces and perform sample acquisition at depths of meters is critical for future NASA in-situ exploration missions to bodies in the solar system, including Mars, Europa, and Enceladus. A corer/sampler was developed with the goal of acquiring pristine samples by reaching depths on Mars beyond the oxidized and sterilized zone. The developed rotary-hammering coring drill, called Auto-Gopher, employs a piezoelectric actuated percussive mechanism for breaking formations and an electric motor rotates the bit to remove the powdered cuttings. This sampler is a wireline drill that is incorporated with an inchworm mechanism allowing thru cyclic coring and core removal to reach great depths. The penetration rate is optimized by simultaneously activating the percussive and rotary motions of the Auto-Gopher. The percussive mechanism is based on the Ultrasonic/Sonic Drill/Corer (USDC) mechanism, which is driven by a piezoelectric stack, demonstrated to require low axial preload. The AutoGopher has been produced taking into account the lessons learned from the development of the Ultrasonic/Sonic Gopher that was designed as a percussive ice drill and was demonstrated in Antarctica in 2005 to reach about 2 meters deep. A field demonstration of the Auto-Gopher is currently being planned with the objective of reaching as deep as 3 to 5 meters in tufa formation.
\end{abstract}

Keywords: Drilling, Deep drill, Auto-Gopher, USDC, Planetary Sampling, Rotary-hammering drill

\section{INTRODUCTION}

One of the main goals of NASA robotic exploration of the solar system is the search for life, water and potential resources for human exploration missions. The detection of potential biosignatures and biomarkers, for both extant and extinct life forms, requires in-situ acquisition of a sample below the surface. The analysis for biosignatures in the subsurface sample would be one of the major objectives of either an in-situ or sample return missions. Since water is an important requirement for extraterrestrial life "as we imagine it", the exploration target for potential future NASA missions includes bodies that have had, or do have, water near the surface including Mars, Europa, and Enceladus. There are several reasons to require samples that are at depth that is greater than 2 meters including highly oxidizing (Mars), high radiation (Europa) and cold/vacuum (Encleadus) environments where life and water cannot exist. For the past 3 years, a joint JPL and Honeybee study has been underway to develop a prototype wireline coring drill, called the Auto-Gopher, for drilling as deep as 3 to 5 meters. This system can be scaled to virtually any depth and can potentially acquire samples from as deep as hundreds of meters. The drill is designed to acquire both powdered cuttings and cores, where the generated cuttings have fine particle sizes [Blake et al., 2003] that could be used directly by many of the analyzers that were/are being developed for the various potential NASA exploration missions. Further, the acquired cores preserve the subsurface stratigraphy and trapped volatiles providing important scientific information about the subsurface.

The developed penetrator/sampler overcomes challenges that are inherent to other deep drills that include being heavy and require high axial preload. Rather than using a long drill or a mechanism that involves adding components and mass to the penetrator bit in order to reach great depths, the Auto-Gopher produces the borehole cyclically by reeling it down via an active tethered mechanism. Once the coring bit reaches its maximum internally available room/length, the drill is reeled back out of the formed borehole, the core is removed and the coring process is resumed from the deeper borehole that was formed. The Auto-Gopher combines rotary, hammering, and anchoring mechanisms, where the latter provides torque preload against the sidewall of the borehole for the penetration mechanism. Generally, percussion and rotation have long been the preferred methods of penetrating materials and formations. Percussion is very effective in fracturing hard, brittle materials like stone and ceramics, whereas rotation is more effective on soft and/or ductile materials such as wood, plastics, and metals. One advantage of rotary drills is the effective removal of cuttings from the borehole via the flutes on the bit. Percussion fractures the material, but continues to hammer at the powdered cuttings inside the borehole unless they are 
removed. This wasted energy that could go into penetrating the medium limits the depth of penetration. Therefore, combining rotation and percussion produces a highly effective penetration mechanism. Existing hammer-drills produce their hammering either pneumatically or mechanically. While the rotation in the Auto-Gopher is actuated by a set of electromagnetic motors, the hammering is generated by the piezoelectric mechanism called the Ultrasonic/Sonic Driller/Corer (USDC) that was demonstrated to require low axial load for its operation. Recently, a breadboard and fully assembled Auto-Gopher were produced and the latter was tested to reach 2 meters depth. Currently, improvements of the mechanism are underway while plans are being made for conducting field test to reach as deep as 3 to 5 meters in a tufa formation.

\subsection{THE USDC MECHANISM}

Many common mechanisms of sampling require high axial forces and holding torques, consume high power, are inefficient in duty cycling, and they also require heavy equipment. To address these limitations, the JPL's Advanced Technologies Group [http://ndeaa.jpl.nasa.gov] developed the USDC mechanism (Figure 1) [Bar-Cohen et al., 1999; Bao et al., 2003; Bar-Cohen and Zacny, 2009]. This development was followed with many improvements that were disclosed in NASA New Technology Reports and patents [for example, Aldrich et al., 2008; Badescu et al., 2006a; 2006b; Bao et al. 2004; 2010; Bar-Cohen et al. 1999; 2003; 2005; 2008; 2010; BarCohen and Sherrit. 2003a; 2003b; Dolgin et al. 2001; Sherrit et al. 2001; 2002; 2003; 2006; 2006; 2008; 2009; 2010a; 2010b]. The USDC requires low axial force making it attractive for operation in low gravity environments allowing to drill and core hard formations using relatively small preload and low mass hardware. Also, it is driven by either continuous or duty cycling allowing effective use of its driving power. The USDC was demonstrated to: 1) drill ice and various rocks including granite, diorite, basalt and limestone; 2) operate at low and high temperatures; and 3) host integrated sensors for measuring various properties. A series of modifications of the USDC basic configuration led to the development of the Ultrasonic/sonic Rock Abrasion Tool (URAT), the Lab-on-a-drill, Ultrasonic/Sonic Gopher for deep ice drilling, the Auto-Gopher for deep drilling in rocks and regolith, and many others.

The USDC consists of three key components: actuator, free-mass and bit (Figure 1) [Bao et al, 2003], where the actuator acts as a hammering mechanism hitting the free-mass and thus the bit fractures the medium underneath. The actuator is driven by a piezoelectric stack having backing designed to forward the generated impact power and in the front a horn is used for amplifying the induced displacements. The piezoelectric stack is driven in resonance, which is about $20-\mathrm{kHz}$ in the basic configuration, and is held by a stress bolt in compression to prevent fracture during operation. In contrast to typical ultrasonic drills, which have the bit physically connected to the horn, in the USDC the actuator hammers a free flying mass (free-mass) that bounces between the horn tip and the top of the bit converts the ultrasonic impacts to sonic frequency hammering blows. The impacts of the free-mass generate stress pulses at the interface of the bit and the rock. The impact stress pulses propagate and fracture the rock when its ultimate strain is exceeded.

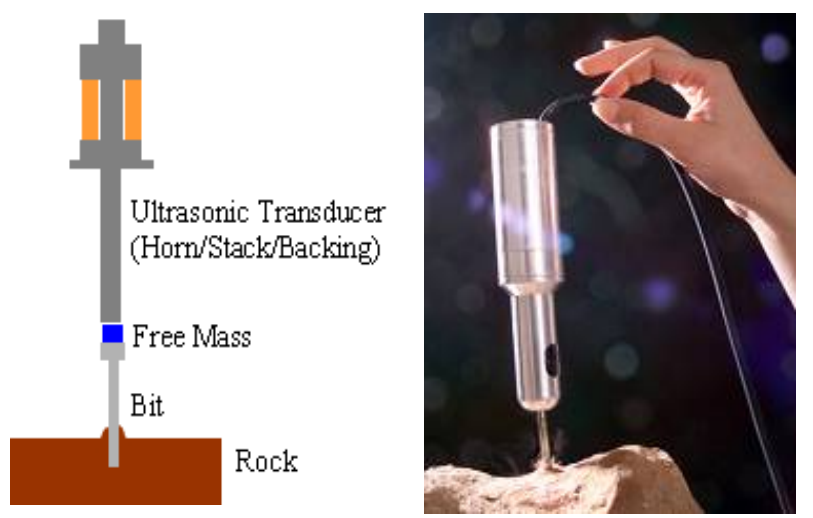

Figure 1: A schematic diagram of the USDC cross-section (left) showing its configuration, and a photograph showing its ability to drill with minimum axial force (right).

\subsection{THE AUTO-GOPHER - BACKGROUND}

The main feature of the Auto-Gopher is its wireline operation [Bar-Cohen et al., 2005; Bar-Cohen and Zacny, 2009]. The drill is essentially suspended on a tether and all the motors and mechanisms are built into a tube that ends with a coring bit (Figure 2). The tether provides the mechanical connection to a rover/lander on a surface as well as the 
power and data communication. Upon reaching the target depth, the drill is retracted from the produced borehole by a pulley system, which can be either on the surface or integrated into a top part of the drill itself.

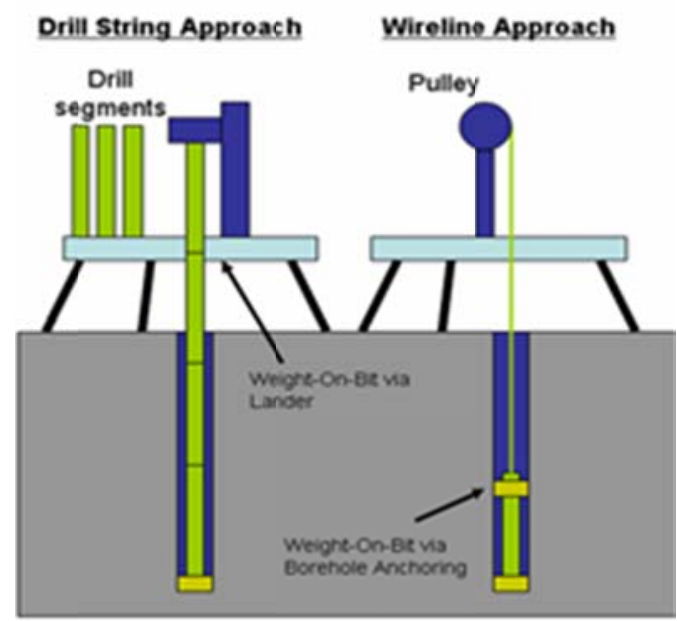

Figure 2: A conventional drill string vs. a wireline drill.

The competing drill designs include the continuous drill string system (Figure 2) where, as the drill gets deeper, new drill sections have to be added. Therefore, this requires drill sections that add greatly to the mass of the system. Also, it requires a drill string feeding mechanism, such as a carousel and mating connections between each drill string, which increases the system complexity. An additional disadvantage of the continuous drill string system is that the power, which is required to convey the cuttings from great depths, will be prohibitively large due to auger drag. The wireline approach solves this problem since the power, required for conveying the sample to a caching chamber located above the drill assembly, is always the same.

Generally, wireline systems involve mechanical complexity of packaging motors and actuators into a slim tube. In addition, as opposed to continuous drill string systems where the Weight on Bit (WoB), also known as preload, is provided by a lander or a rover, the WOB in wireline systems is provided by anchoring the drill to the borehole wall. The anchoring locks the upper section of the drill using an internal screw to push on the drilling mechanism and the drill bit itself. This is an added advantage of the wireline drills since the WoB of the continuous drill system is limited by the weight of the lander/rover itself.

The Auto-Gopher also overcomes challenges that are inherent to deep ice drills including melting or hot-water drills that are used to drill pure ice [Zimmerman, 2002]. The main disadvantage of the prior drills is their high mass and complex fixtures that cannot be carried with a small rover. Also, hot-water drills and other melt probes do not produce core, they require a source of large amount of ultra-clean water, they have high power requirements and they are difficult to operate in ice with sediments or permafrost, or when large rocks are present.

Other, non-traditional drilling technologies (laser, electron beam, microwave, jet, etc.) usually are competitive only in applications that are time limited and not power/mass limited as is typical for space science applications. Generally, future space missions would not have enough power (or rather electrical energy) to employ these "modern" drilling technologies. In contrast, the developed low mass Auto-Gopher uses low power and low WOB/preload, and it is not constrained by the mass of a lander/rover to penetrate a formation and acquire cores. Acquired cores would retain the stratigraphy (and volatiles if present) to provide significant scientific information about the layered structure with inclusions and potential organisms, as well as contain trapped volatiles and their valuable information.

The main disadvantage of the wireline system is a possibility of borehole collapse. However, since the developed drill will be deployed in ice or ice-cement ground, the risk is low at depths of less than $20 \mathrm{~m}$.

\subsection{THE BREADBOARD OF THE AUTO-GOPHER}

The first step in producing the Auto-Gopher as a wireline unit consisted of fabricating a breadboard with a motor rotating the bit from the side. This unit allowed the development and optimization of the performance of the piezoelectric actuator that is to be used to drive percussive mechanism of the Auto-Gopher. The bit was made of aluminum onto which a steel crown ring, with brazed tungsten carbide teeth, was screwed. The bit was designed with flutes to propel the cuttings upward out of the formed borehole and at the top end of the flute a hole was drilled to provide a window for entry of the powdered cuttings into the chamber above the formed core. This removed the 
powder from the drilled surface and allows for collection of cuttings sample for subsequent analysis. To test the performance of the breadboard a testbed was constructed that allows for controlling the preload and the drive parameters of the drill (speed, power, etc.). In addition, the drill was mounted onto a slider to allow the whole assembly to move freely in the drilling direction. In parallel, using Matlab and Labview software was developed that allows for modeling the dynamics of various configurations of the USDC and several designs were tested. The results were used to optimize the design of the integrated Auto-Gopher. A photo of the breadboard mounted onto the testbed and an illustration of the coring bit cross-section are shown in Figure 3.
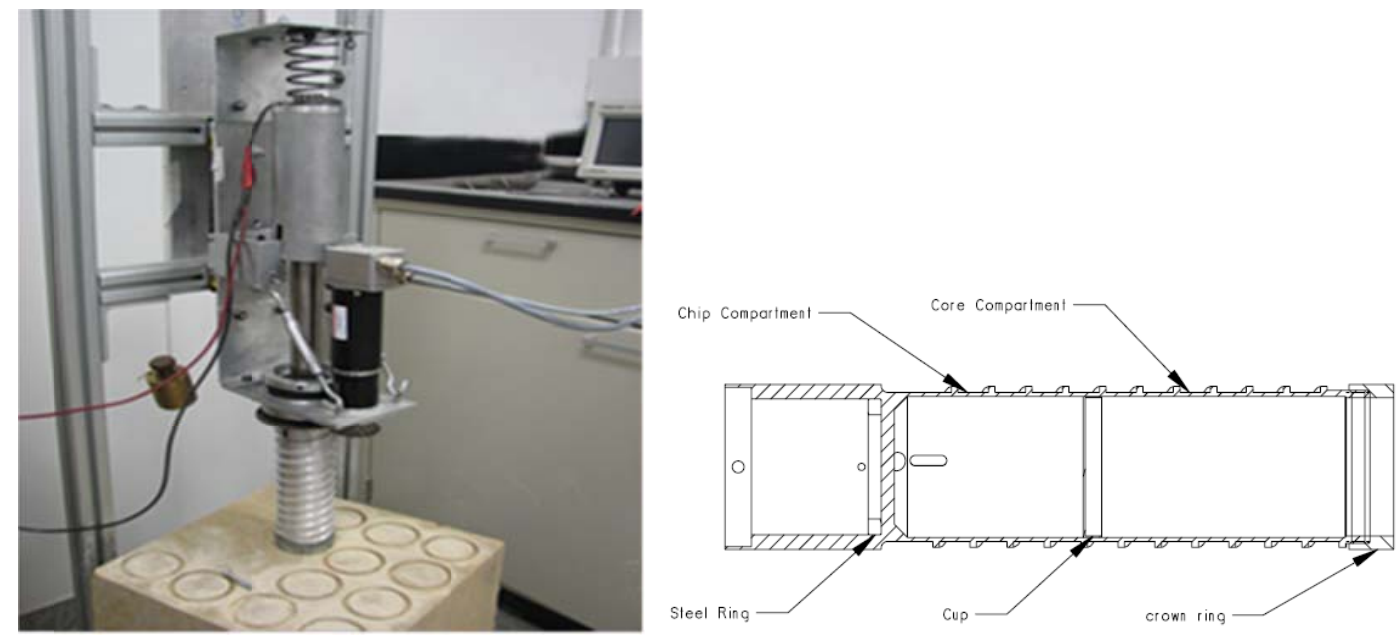

Figure 3: The breadboard on the testbed and a cross-section the coring bit

\subsection{THE FABRICATION AND TESTING OF THE AUTO-GOPHER}

Using the results of the analysis, fabrication and testing of the breadboard, the full size Auto-Gopher was designed and produced (Figure 4). This fully assembled Auto-Gopher employs a piezoelectric actuated percussive mechanism for providing impacts and a cluster of 3 motors are used for rotating the coring bit with auger. This wireline drill allows coring and core removal from depths limited only by the length of a deployment tether.

The outside diameter of the coring bit is $72 \mathrm{~mm}$. The length of the drill is $2 \mathrm{~m}$ and it weighs $20 \mathrm{~kg}$. The powdered cuttings are moved up the auger flutes and fall into the cuttings chamber (i.e., chip compartment) above the core chamber. Upon reaching a core length of $10 \mathrm{~cm}$, the Auto-Gopher is retracted and the cuttings chamber is emptied and the core is removed. In order to reduce the drill complexity and the risk of having the drill getting stuck if the core cannot be sheared, the Auto-Gopher currently does not have core catching capabilities. However, future generation of the Auto-Gopher will be developed with an auto-core catching feature employing core-dogs or other core break off mechanism [Badescu et al., 2009a; Badescu et al., 2009b; Badescu et al., 2009c]. The drill uses a set of three plates to push against the wall of the borehole and anchors itself. The WoB is provided by internally actuated ball screw and an integrated load cell provides a force feedback.

The system level testing of the Auto-Gopher was performed in a 2 meter block of Texas Limestone having strength of $25 \mathrm{MPa}$ (Figure 5). We performed two different tests of reaching a 2 meter depth. In the first test, we used the rotary-only mode of drilling, while during the $2^{\text {nd }}$ round of tests, we used the full rotary-percussive drilling capability where the percussion was piezo-driven by the USDC mechanism.

During the rotary-only test, the average power was 90 Watt at $25 \%$ efficiency, i.e. the power required to drill was 25 Watts while the rest was attributed to electrical/mechanical losses. The rotational speed was $90 \mathrm{rpm}$ and it took 15 minutes to drill $10 \mathrm{~cm}$ long core (i.e. penetration rate was $40 \mathrm{~cm}$ per hour). Drilling to 2 meter depth and recovering of cores every $10 \mathrm{~cm}$ took a total time of 15 hours (a single step of drilling $10 \mathrm{~cm}$ and retrieving the core was 45 minutes). A photo of the formed core is shown in Figure 6. Total energy to reach the $2 \mathrm{~m}$ depth was 500 W.hr. The Weight on Bit was limited to less than 70 Newton. The cores recovery was $100 \%$ successful.

When the piezo-actuated percussive system was turned-on the required auger power decreased by $20 \%$. It is probably due to the reduction of the torque that is required to move the cuttings up the auger and the reduction of auger drag. The piezo system was vibrated at $5 \mathrm{kHz}$ and it required approximately 60 Watts of power. It is interesting to note that the cuttings were packed in the cuttings chamber and, hence, this cuttings chamber can be made smaller in the next generation system. 


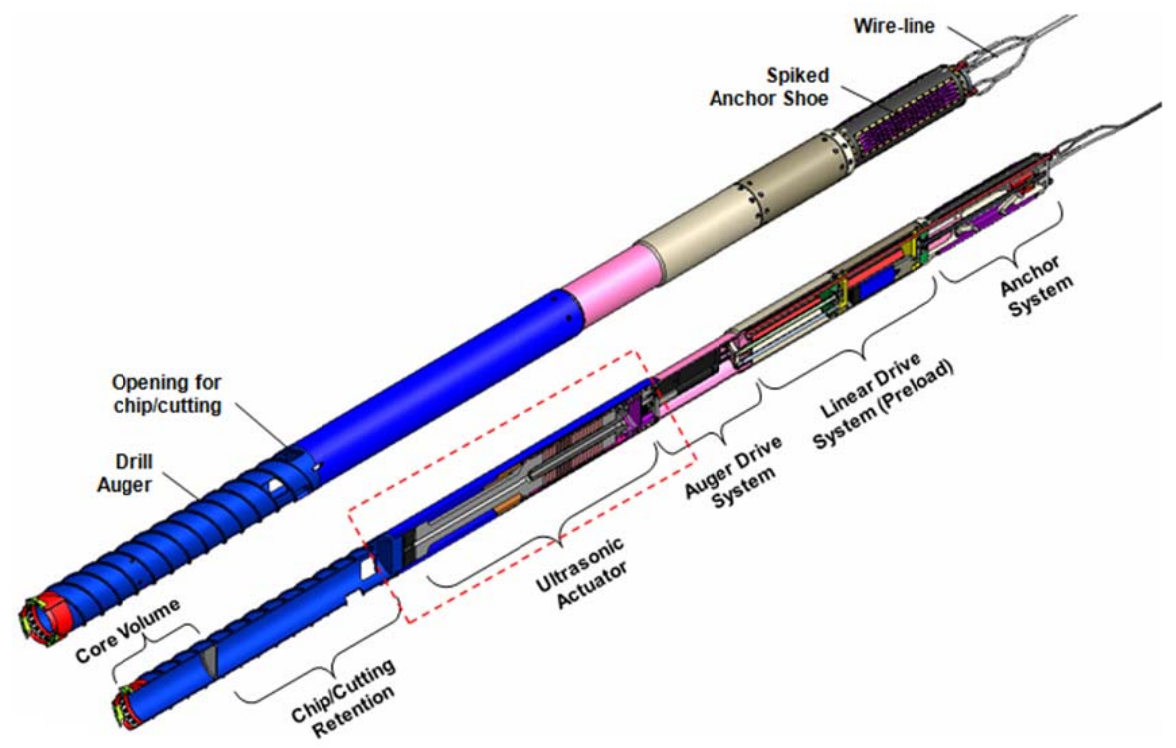

Figure 4: The components of the Auto-Gopher.

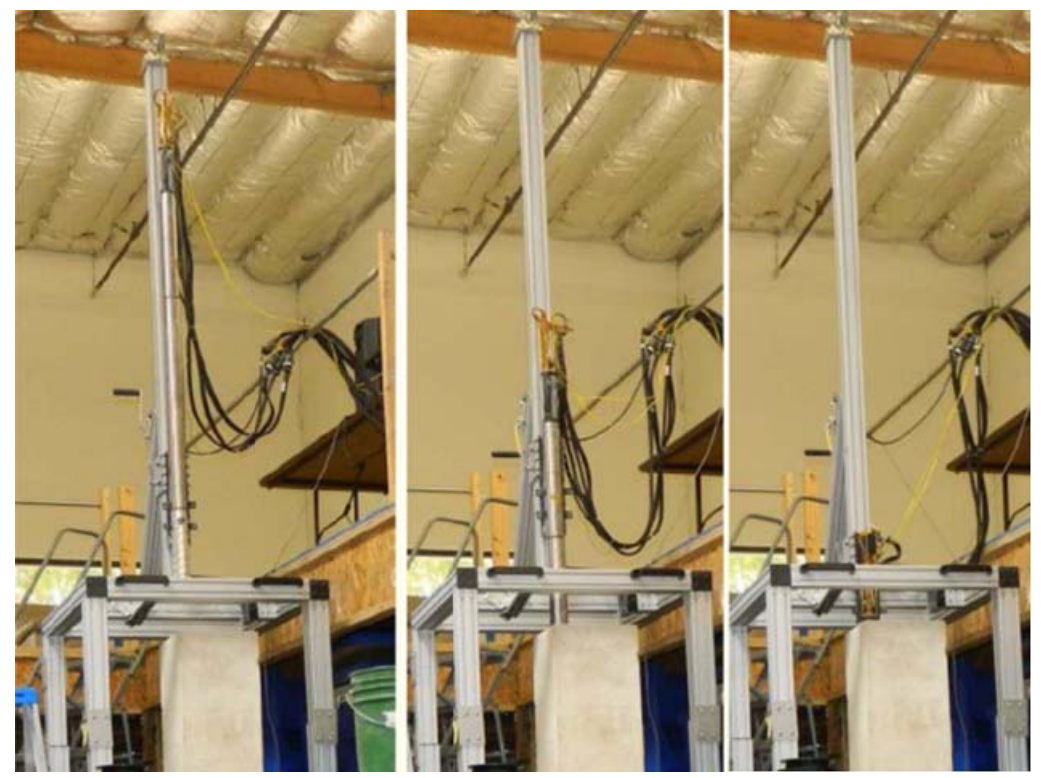

Figure 5: Drilling progress into 2 meter high limestone column.

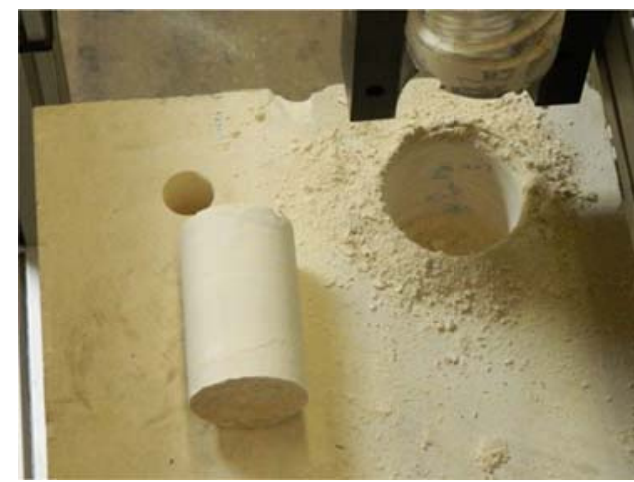

Figure 6: The recovered core samples were $57 \mathrm{~mm}$ diameter and $100 \mathrm{~mm}$ long. The cores recovery was $100 \%$ successful. 


\section{SUMMARY}

An Auto-Gopher, which is a wireline drill, was developed for deep drilling in potential future in-situ exploration missions. The drill operates cyclically coring, uploading the acquired core, downloading the drill into the borehole, and repeating the process. The design of the Auto-Gopher was based on lessons learned from the operation of an ice drill version that was demonstrated in Antarctica reaching about 2-meter deep. The drill is a rotary-hammering mechanism that combines rotation by a motor and percussive actuation by the ultrasonic/sonic driller/corer (USDC) mechanism. The latter is a drilling technique that requires low axial preload and has been the subject of extensive studies at JPL. To allow effective design of the USDC, it was analytical modeled to predict its performance and produce a drill with optimized performance. A breadboard and fully assembled Auto-Gopher units were produced and tested. The latter was demonstrated to reach 2-meters deep in limestone using both rotary-only and rotation and percussive drilling, where the addition of the percussion was observed to reduce the required auger power by $20 \%$. While the capability of the developed Auto-Gopher is currently being improved, plans are underway to field test it to reach 3 to 5 meters deep in tufa formation.

\section{ACKNOWLEDGEMENT}

Research reported in this manuscript was conducted at the Jet Propulsion Laboratory (JPL), California Institute of Technology, under a contract with the National Aeronautics and Space Administration (NASA). This task was funded by the NASA Science and Technology for Exploring Planets (ASTEP) program.

\section{REFERENCES}

Aldrich J., Y. Bar-Cohen, S. Sherrit, M. Badescu, X. Bao, and J. Scott, "Percussive Augmenter of Rotary Drills (PARoD) for Operating as a Rotary-Hammer Drill,” NTR Docket No. 46550, (2008).

Badescu M., S. Sherrit, A. Olorunsola, J. Aldrich, X. Bao, Y. Bar-Cohen, Z. Chang, P. T. Doran, C. H. Fritsen, F. Kenig, C. P. McKay, A. Murray, S. Du, T. Peterson, and T. Song, "Ultrasonic/sonic Gopher for subsurface ice and brine sampling: analysis and fabrication challenges, and testing results," Proceedings of the SPIE Smart Structures and Materials Symposium, Paper \#6171-07, San Diego, CA, (2006a).

Badescu M., S. Sherrit, Y. Bar-Cohen, X. Bao, and S. Kassab, "Ultrasonic/Sonic Rotary-Hammer Drill (USRoHD)," U.S. Patent No. 7,740,088, June 22, 2010. NASA New Technology Report (NTR) No. 44765, (2006b).

Badescu M., D. B. Bickler, S. Sherrit, Y. Bar-Cohen, X. Bao, and N. H. Hudson, "Rolling tooth core break-off and retention mechanism.” NTR Docket No. 47354, Submitted on (October 19, 200a9). NASA Tech Briefs, Vol. 35, No. 6, 2011, pp. 50-51.

Badescu M., S. Sherrit, Y. Bar-Cohen, X. Bao, and P. G. Backes, "Scoring dawg - core break-off and retention mechanism,” NTR Docket No. 47355, Submitted on (October 19, 2009b). NASA Tech Briefs, Vol. 35, No. 6, 2011, pp. 48-49.

Badescu M., S. Sherrit, Y. Bar-Cohen, X. Bao, and R. A. Lindemann, "Praying mantis - bending core break-off and retention mechanism, NTR Docket No. 47356, Submitted on (October 19, 2009c). NASA Tech Briefs, Vol. 35, No. 6, 2011, pp. 49-50.

Bao X., Y. Bar-Cohen, Z. Chang, B. P. Dolgin, S. Sherrit, D. S. Pal, S. Du, and T. Peterson, "Modeling and Computer Simulation of Ultrasonic/Sonic Driller/Corer (USDC),"IEEE Transaction on Ultrasonics, Ferroelectrics and Frequency Control (UFFC), Vol. 50, No. 9, (2003), pp. 1147-1160.

Bao X., Y. Bar-Cohen, Z. Chang, S. Sherrit and R. Stark, "Ultrasonic/Sonic Impacting Penetrator (USIP),” NASA NTR No. 41666 (2004).

Bao X., S. Sherrit, M. Badescu, Y. Bar-Cohen, S. Askins, and P. Ostlund, "Free-mass and interface configurations of hammering mechanisms,” Patent was filled on October 27, 2011, NTR Docket No. 47780 (2010).

Bar-Cohen Y., S. Sherrit, B. Dolgin, T. Peterson, D. Pal and J. Kroh, "Smart-ultrasonic/sonic driller/corer," U.S. Patent No. 6,863,136, March 8, 2005, NASA NTR No. 20856 (1999)

Bar-Cohen Y. and K. Zacny (Eds.), "Drilling in Extreme Environments - Penetration and Sampling on Earth and Other Planets,” Wiley - VCH, Hoboken, NJ, ISBN-10: 3527408525, ISBN-13: 9783527408528, (2009) pp. 1827.

Bar-Cohen Y., S. Sherrit and J. L. Herz "Ultrasonic/Sonic Jackhammer (USJ),” NASA New Technology Report (NTR), Docket No. 40771 (2003).

Bar-Cohen Y., and S. Sherrit, "Self-Mountable and Extractable Ultrasonic/Sonic Anchor (U/S-Anchor)," NASA NTR No. 40827 (2003a).

Bar-Cohen Y., and S. Sherrit, "Thermocouple-on-the-bit a real time sensor of the hardness of drilled objects," NASA NTR No. 40132 (2003b) 
Bar-Cohen Y., S. Sherrit, B. Dolgin, X. Bao and S. Askin, "Ultrasonic/Sonic Mechanism of Deep Drilling (USMOD)," U.S. Patent No. 6,968,910, (2005).

Bar-Cohen Y., M. Badescu, and S. Sherrit, "Rapid Rotary-Percussive Auto-Gopher for deep subsurface penetration and sampling,” NASA NTR No. 45949 (2008).

Bar-Cohen Y., M. Badescu, and S. Sherrit, "Acquisition and retaining granular samples via rotating coring bit," NASA NTR No. 47606 (2010).

Blake D.F., P. Sarrazin, S. J. Chipera, D. L. Bish, D. T. Vaniman, Y. Bar-Cohen, S. Sherrit, S. Collins, B. Boyer, C. Bryson and J. King, "Definitive Mineralogical Analysis of Martian Rocks and Soil Using the CHEMIN XRD/XRF Instrument and the USDC Sampler,” Proceedings of the Sixth International Conference on Mars, held at Caltech, Pasadena, CA, July 20-25, 2003.

Dolgin B., S. Sherrit, Y. Bar-Cohen, R. Rainen, S. Askins and D. Sigel, D. Bickler, J. Carson, S. Dawson, X. Bao, and Z. Chang, and T. Peterson, "Ultrasonic Rock Abrasion Tool (URAT)," NASA NTR No. 30403 (2001).

Sherrit S., S. A. Askins, M. Gradziel, B. P. Dolgin, Y. Bar-Cohen, X. Bao, and Z. Cheng, "Novel ultrasonic horns for power ultrasonics, ” NASA Tech Briefs, Vol. 27, No. 4, 2003, pp. 54-55, NASA NTR No. 30489 (2001)

Sherrit S., Y. Bar-Cohen, B. Dolgin, X. Bao, and Z. Chang, "Ultrasonic Crusher for Crushing, Milling, and Powdering," NASA NTR No. 30682 (2002).

Sherrit S., Y. Bar-Cohen, X. Bao, Z. Chang, D. Blake and C. Bryson, "Ultrasonic/Sonic Rock Powdering Sampler and Delivery Tool,” NASA NTR No. 40564 (2003)

Sherrit S., M. Badescu, Y. Bar-Cohen, Z. Chang, X. Bao, "Portable Rapid and Quiet Drill (PRAQD)," Patent disclosure submitted on Feb. 2006. U.S. Patent No. 7,824,247, November 4, 2010. NASA NTR No. 42131 (2005).

Sherrit S., Y. Bar-Cohen, M. Badescu, X. Bao, Z Chang, C. Jones, J. Aldrich, "Compact Non-Pneumatic Powder Sampler (NPPS),” NASA NTR No. 43614 (2006).

Sherrit S., M. Badescu, and Y. Bar-Cohen, "Miniature Low-Mass Drill Actuated by Flextensional Piezo-Stack (DAFPiS)” NASA NTR No. 45857, (2008).

Sherrit S., X. Bao, M. Badescu, and Y. Bar-Cohen, "Single Piezo-Actuator Rotary-Hammering (SPaRH) Drill," NASA NTR No. 47216, (2009).

Sherrit S., X Bao, M. Badescu, Y. Bar-Cohen, and P. Allen, "Monolithic Flexure Pre-stressed Ultrasonic Horns,” A Provisional Patent Application 61/362,164 was filed on July 8, 2010. NASA NTR No. 47610 (2010a).

Sherrit S., X. Bao, M. Badescu, Y. Bar-Cohen, P. Ostlund, P. Allen, and D. Geiyer, "Planar Rotary Piezoelectric Motor using Ultrasonic Horns," A Provisional Patent was filed on July 7, 2011, NASA NTR No. 47813 (2010b).

Zimmerman et al., “The Mars '07 North Polar Cap Deep Penetration Cryoscout Mission,” IEEE Aerospace Conf. (2002) 